\title{
Chemistry and Structure of Graphene Oxide via Direct Imaging
}

Shreya H. Dave

Department of Mechanical Engineering, Massachusetts Institute of Technology sdave@mit.edu

Chuncheng Gong

Department of Materials, University of Oxford

chuncheng.gong@,materials.ox.ac.uk

Alex W. Robertson

Department of Materials, University of Oxford

alex.robertson2@materials.ox.ac.uk

Jamie H. Warner

Department of Materials, University of Oxford

jamie.warner@materials.ox.ac.uk

Jeffrey C. Grossman*

Department of Materials Science \& Engineering, Massachusetts Institute of Technology

77 Massachusetts Avenue, Room 13-5049

Cambridge, MA 02139 USA

617-324-3566

jcg@mit.edu

*indicates corresponding author 


\section{ABSTRACT}

Graphene oxide (GO) and reduced GO (rGO) are the only variants of graphene that can be manufactured at the kilogram scale, and yet the widely accepted model for their structure has largely relied on indirect evidence. Notably, existing HRTEM studies of graphene oxide report long-range order in relatively defect-free GO. Here, we present the first HRTEM evidence of the Dynamic Structural Model, in which GO is progressively degraded into a nanocrystalline structure. Direct observation of the intrinsic atomic structure of this defective GO has until now been inhibited by the presence of contaminants that adsorb to the surface of the material at room temperature. We use an in-situ heating holder within an aberration-corrected low-voltage TEM to study the atomic structure of graphene oxide from room temperature to $700{ }^{\circ} \mathrm{C}$. As the temperature increases to above $500{ }^{\circ} \mathrm{C}$, the adsorbates detach from the $\mathrm{GO}$ and the underlying atomic structure is imaged to be small $2-4 \mathrm{~nm}$ crystalline domains within a polycrystalline GO film. By combining spectroscopic evidence with the HRTEM data, we support the dynamic interpretation of the structural evolution of graphene oxide.

\section{BODY}

GO is the most readily scalable derivative of graphene, significantly less expensive and considerably more solution processable when compared to CVD graphene and mechanical exfoliation, respectively. GO is formed by heavily oxidizing graphite to chemically exfoliate the flakes of the graphitic stack into mono- and few-layer sheets, depending on the degree of oxidation and post-processing. Graphite oxide was first prepared by Brodie in $1859^{1}$ though many commercially implemented methods today rely modifications to the Hummers Method ${ }^{2-4}$. GO is notoriously difficult to characterize and define broadly because of its inherent non- 
stoichiometric structure and dependence on production parameters. Dimiev et al. have proposed a Dynamic Structural Model (DSM) for GO in which the carbon lattice is progressively degraded upon exposure to water or a strong base ${ }^{5,6}$. In their model, GO reacts with water to explain its acidic nature through two transformations: (1) the development of oxygen functionalities; and (2) the conjugation of aromatic domains.

Work on both graphene ${ }^{3}$ and $\mathrm{GO}^{7}$ has revealed the presence of hydrocarbons that weakly attach (physioadsorb) to the surface of the material, particularly in defective areas. Recently, there has been interest in the presence and characterization of surface contaminants at ambient conditions on both graphene and graphene oxide $(\mathrm{GO})^{2,8-12}$. These contaminants affect the surface properties: increasing the wettability of the graphene $e^{9,13,13,14}$ or potentially decreasing the conductivity of graphene oxide ${ }^{2}$. Further, the surface contaminants pose a challenge to characterizing the intrinsic properties of the material, including its chemical structure, nature of order and disorder, and defect density, particularly for GO whose structure has been the subject of a number of different, at times conflicting, theories ${ }^{2,9,13-17}$. In order for graphene or GO to achieve a "killer app", both these contaminants and the inherent structural transformation of GO must be fully understood $2,7,8,8-12,15,18-20$

While direct imaging of the atomic structure of graphene has been achieved extensively using aberration-corrected high-resolution transmission electron microscopy (HRTEM), it has proven more challenging to apply similar approaches to GO, particularly more defective GO, due to the reaction of the surface contaminants with the electron beam. Recent work has revealed some insights into the atomic structure of relatively defect-free and intact GO by $\operatorname{HRTEM}^{7,15,18,21}$. 
Meanwhile, methods such as baking in air ${ }^{15,22,23}$, vacuum ${ }^{3,24}$, or carbon black $^{13,21,25}$ reliably clean the graphene for direct observation using microscopy, but our experiments find that none of these methods are effective in cleaning GO or rGO. We attribute this difference to the presence of defects that have a greater tendency to adsorb airborne contaminants.

GO is characterized by oxygen functionalization on $20-30 \%$ of the basal plane, limiting its electrical and thermal transport and thus its widespread use in electronics and optical applications ${ }^{7,15,22,23,26}$. Although multiple models for the structure of GO have been $\operatorname{proposed}^{24,27}$, the most widely accepted Lerf-Klinowski model reports the decoration of the carbon basal plane with epoxides and hydroxyls, with sheet edges terminated by carboxylic acid functional groups ${ }^{9}$. The oxygen-containing functionalization disrupts the $\mathrm{sp}^{2}$ order of the graphene backbone such that regions of $\mathrm{sp}^{2}$ (ordered) are interrupted by the $\mathrm{sp}^{3}$ (disordered) in the carbon basal plane $e^{2,10,13,25}$. Meanwhile, direct observation of GO using transmission electron microscopy (TEM) showed the structure of GO as consisting of long-range ordered $\mathrm{sp}^{2}$ regions, and characterized by much smaller patches of amorphous material and/or defects, which cannot fully account for the functionalized regions.

Processing GO to remove oxygen functional groups has been thoroughly explored with both chemical $^{23,28}$ and thermal ${ }^{2,9-12,24,25,29}$ and simulation ${ }^{8,9,20}$ methods. These methods optimize the reduction parameters such that there is sufficient oxygen removal without considerable damage to the graphitic sheet; in other words, removing more oxygen is favorable for conductivity improvements, but lattice vacancies destroy the integrity of the GO flake, limiting its conductivity. Thermograviometric analysis (TGA) combined with mass spectrometry (MS) 
shows the removal of $\mathrm{CO}$ and $\mathrm{CO}_{2}$ during thermal annealing, and attributes vacancies in the lattice structure to the source of carbon $2,13,14,16$.

Here, we present a study of the atomic structure of commercially available GO, which exhibits short-range order that is likely a result of degradation in accordance with the Dynamic Structural Model. As the first direct evidence of the DSM, we note that the mechanisms proposed by Dimiev and colleagues play a significant role in the development of commercial applications for graphene oxide. We characterize the GO with spectroscopic methods to confirm that the indirect evidence is consistent with a large range of literature. GO of this nature is unilaterally characterized by a layer of amorphous contaminants, though not a result of oxidation as suggested by Rourke and colleagues ${ }^{2,10}$ because in our studies their presence is reversible with temperature cycles. We report on the behavior of the surface contaminants in the vacuum of the TEM and present a method to remove them in order to achieve atomic resolution of GO structure. Finally, we confirm that the structure of highly defective GO resembles the SzaboDekany Model ${ }^{23,27,28}$, in which the structure of GO consists of nano-crystallines and disrupted by grain boundaries of $\mathrm{sp}^{3}$ bonds.

\section{Results \& Discussion}

Surface contaminants We conducted a repeatable study of the structure of graphene oxide using aberration-corrected high-resolution TEM at 80kV (AC-TEM). Knock-on damage for pristine graphene is reported to occur at $\sim 84 \mathrm{kV}^{19,30}$ and thus graphene and its derivatives are observed at low voltage with spherical aberration (Cs) correction in order to resolve the atomic structure ${ }^{2,13,17,31,32}$. We prepared samples on heating chips for TEM (DENs) with a platinum 
coil shown in Figure 1, a. Slits were introduced into the silicon nitride thin film using a focused ion beam to allow the GO to be suspended across vacuum for enhanced contrast and atomic resolution imaging, shown in Figure 1, b. Macroscopically, GO is wrinkled and appears clumped consistently through multiple samples, shown in Figure 1, c-d.

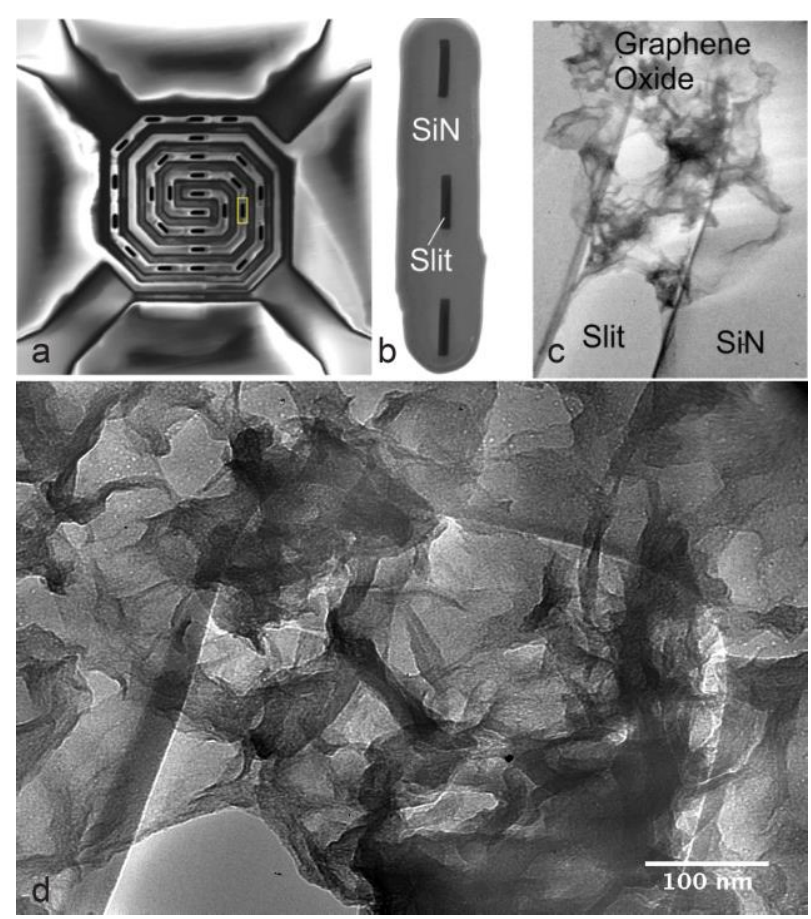

Figure 1 Low-voltage, high-resolution TEM was used to directly observe the structure of graphene oxide. A heating chip containing a platinum coil (a) is used to study the temperature dependence of the structure. A slit was introduced into the thin silicon nitride membrane using a focused ion beam (FIB) (b) in order to obtain suspended GO. Low-magnification images (c-d) show the macroscopic morphology of graphene oxide: clumped flakes with monolayer regions.

At room temperature and in the high vacuum of the TEM, the structure of GO visually appears fully amorphous and decomposes rapidly under the energy of the beam (see Supplementary Figure 1). This interaction of contamination and the beam is not surprising and was observed in a number of different sample preparation methods, as well as different solvents, graphene oxide feedstocks, and ambient conditions of preparation. It is possible that the relative quantity of 
amorphous contaminants could be affected by the presence of the electron beam, however we are not the first to observe such adventitious carbon in the GO system ${ }^{7,15,26,33}$. Numerous experiments utilizing cleaning methods reported for other two-dimensional materials were conducted and were unable to clear the contamination from GO for imaging $\mathrm{g}^{3,13,15,21-25}$ leading us to believe that surface contaminants tend to be strongly attached to the GO sample than to twodimensional materials with longer-range order. At temperatures above $500{ }^{\circ} \mathrm{C}$, the specimen appears more crystalline and we are able to resolve the atomic structure of GO. In Figure 2 a, we show a region of graphene oxide in false color to enhance contrast. Both open (monolayer, low contrast) and closed (folded over bilayer, high contrast) edges are visible as are $\mathrm{sp}^{3}$ regions that are separated by rotation relative to each other. The high contrast of the closed edges is different from heavier atoms contaminating the sample (Supplementary Figure 2). Figure $2 b$, shows the atomic structure of a region of monolayer $\mathrm{GO}$ at a temperature of ?, with sufficient resolution to identify defects (pentagons and heptagons) in the hexagonal $\mathrm{sp}^{2}$ lattice.
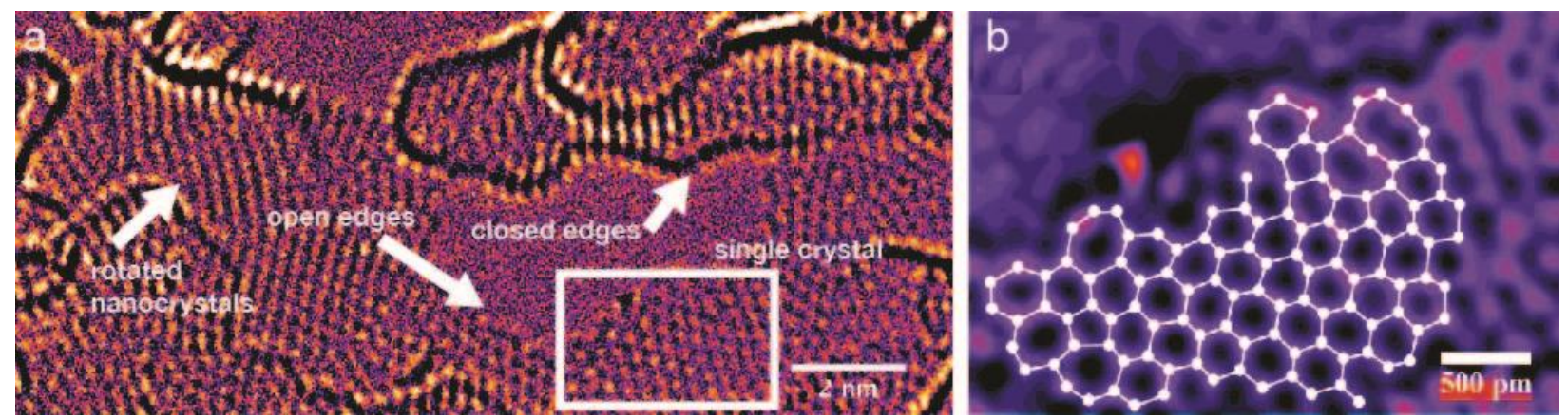

Figure 2 Atomic structure can be resolved using in-situ heating with AC-TEM with features shown in (a). Sufficient resolution is achieved to identify defects in the bond structure (b). In (b) not all the atomic structure could be accurately resolved, likely due to structural changes occurring during image acquisition and only the directly resolved atomic structure is indicated by white dots and lines. 
At room temperature, this atomic resolution is not visible. Figure 3 shows the comparison of GO at room temperature (a-c) and at $700{ }^{\circ} \mathrm{C}(\mathrm{d}-\mathrm{f})$. The FFTs for different regions are shown, with the characteristic pattern of $\mathrm{sp}^{2}$ graphene lattice (spots at $1 / 20 \mathrm{~nm}^{-1}$ ) observed at $700{ }^{\circ} \mathrm{C}$ for small regions. The insets in Figure $3 \mathrm{a}$ and $3 \mathrm{~d}$ show region iii in each to demonstrate the shift from amorphous contaminants to visible atomic structure.
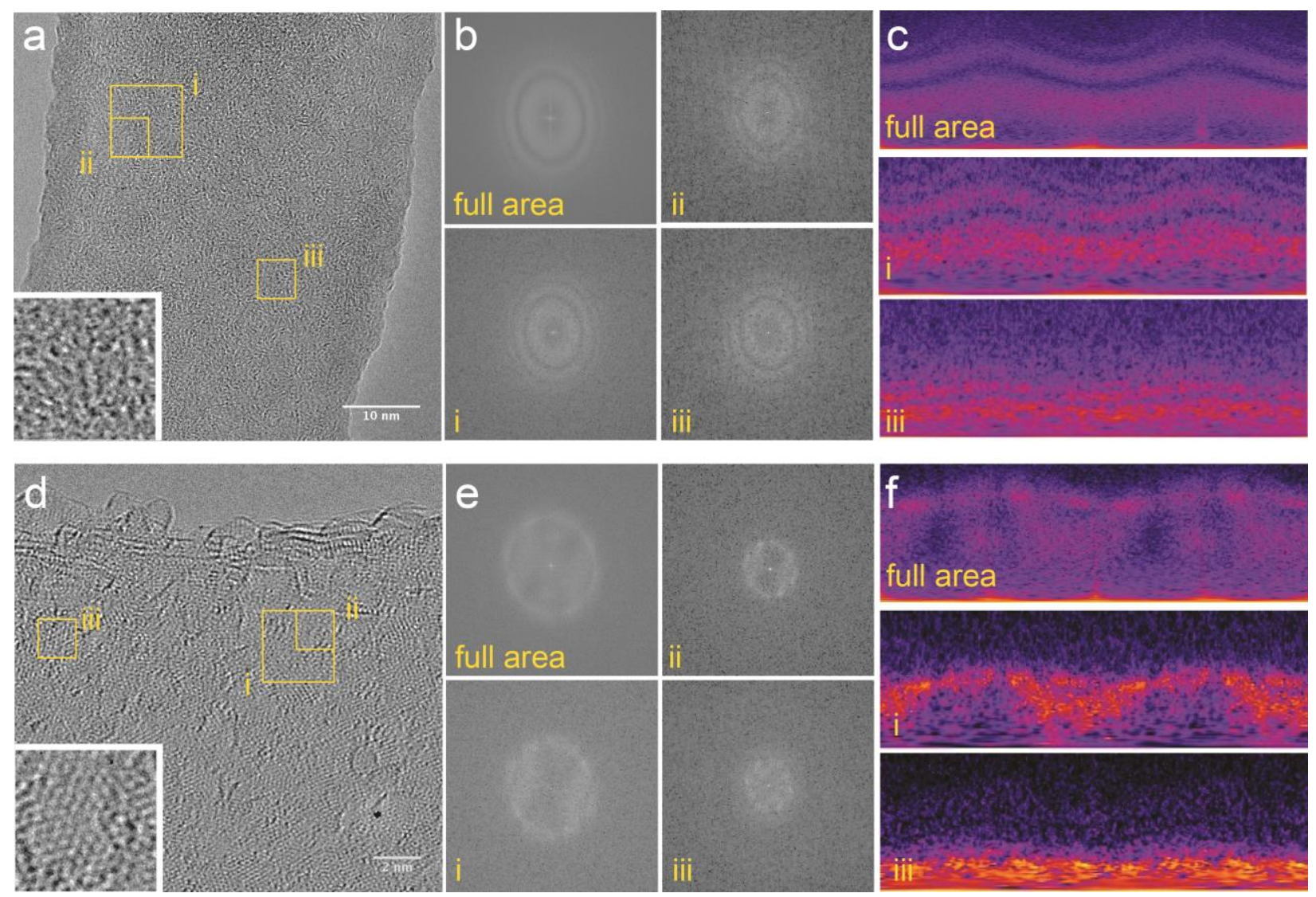

Figure 3 Graphene oxide observed at room temperature (a-c) and $700{ }^{\circ} \mathrm{C}(\mathrm{d}-\mathrm{f})$ in $\mathrm{AC}$-TEM is notably different in appearance. (a) and (d) show images of the material at room temperature and $700{ }^{\circ} \mathrm{C}$ respectively, with insets of the $5 \mathrm{~nm} \times 5 \mathrm{~nm}$ region iii magnified for each. (b) and (e) show FFTs, both of which have a strong band at $1 / \mathrm{d}=1 / 0.21 \mathrm{~nm}^{-1}$ suggesting polycrystalline graphene domains. (c) and (f) polar transforms of the FFTs showing increased order with decreased analysis size. Both the image and the FFTs show high amounts of disorder or amorphous material at room temperature. At $700{ }^{\circ} \mathrm{C}$, although the full region FFT appears similarly amorphous, analysis of smaller components shows the distinct pattern of $\mathrm{sp}^{2}$ graphene lattice, even in multilayer regions. 
We believe that this disorder is a result of surface contaminants rather than oxygen functionalization for two reasons. First, the specimen appears more crystalline above $500{ }^{\circ} \mathrm{C}$, which is consistent with previous reports of amorphous hydrocarbon adsorbates on the surface of CVD graphene ${ }^{4,34}$ and with the observation of adventitious carbon on $\mathrm{GO}^{2,15,18}$. The relative amount of surface contaminants is not defined (as compared to oxide on metals or silicon) but appears to correlate with degree of long range order as previous HRTEM of GO reports have considerably more visible lattice ${ }^{7,15}$ and ssNMR on lab samples with long-range order do not show large amounts of adsorbed amorphous material ${ }^{7,15,35}$.

Second, the sample again appears amorphous upon cooling to room temperature, suggesting that the contaminants have returned (Figure 4). This has been observed in graphene, but graphene tends to retain areas of visible crystal structure whereas samples of GO appear qualitatively amorphous throughout ${ }^{34}$. In both cases, the surface contaminants return even in the high vacuum and anti-contamination protected environment of the TEM, suggesting that their origin is not strictly the oxidation process but instead associated with compounds in the environment, i.e. not the same oxidative debris (OD) reported by Rourke ${ }^{2,36}$.

Figure 4 a shows a region imaged at room temperature after first heating to $700{ }^{\circ} \mathrm{C}$. The inset shows the region within the yellow box. The relatively light contrast areas are a result of beam damage and continue to grow with increased beam exposure. Figure $4 \mathrm{~b}$ shows regions of a cooled sample with their associated FFTs in Figure $4 \mathrm{c}$. Figure $4 \mathrm{~d}$ shows the polar transforms of each of the four regions, decreasing in size, to show the lack of lattice structure shown previously in contrast to Figure $3 \mathrm{f}$. These results indicate that any form spectroscopy conducted at ambient 
temperature necessarily measures both the contaminants and the intrinsic GO structure. Although it is as of now, not possible to fully decouple the groups associated with the surface contaminants and the functional groups bonded directly to the basal plane of the GO, we remark on the importance of this observation in any processing, functionalization, or characterization analysis of GO at ambient temperature, even in vacuum.
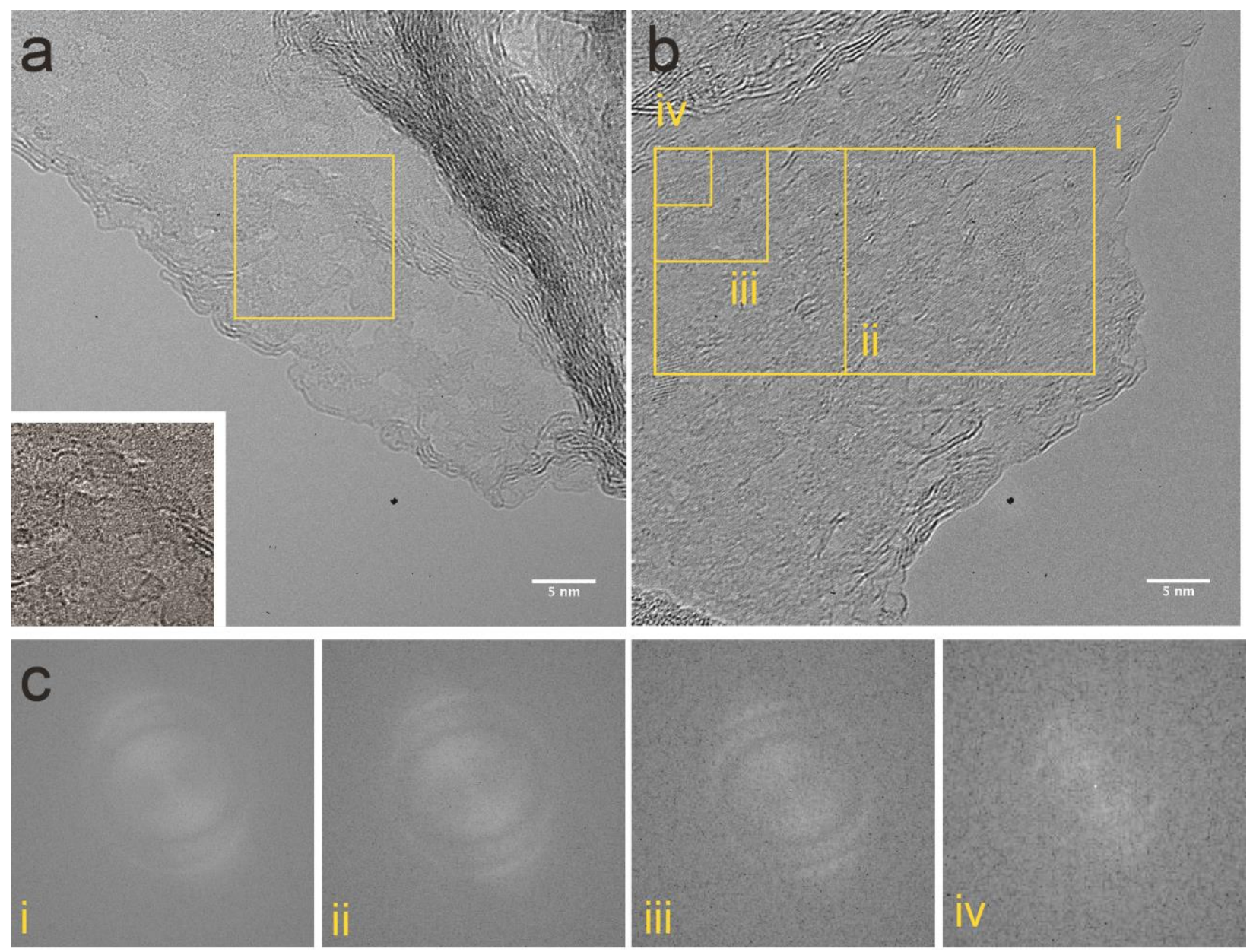

Figure 4 Two different samples are shown to be amorphous when cooled from $700{ }^{\circ} \mathrm{C}$ to room temperature inside the TEM. (a) shows a region with beam damage caused by exposure at room temperature; regions of lighter contrast in the inset are patches of damage that grow with prolonged exposure. The boxed areas in (b) are analyzed with the FFTs in (c). 
Nanocrystalline GO The use of in-situ heating to image GO presents a new opportunity to directly observe the crystal structure of defective GO with atomic resolution. We report on the microstructure of this commercially available GO in order to provide structural information about large-batch GO production. According to the DSM, GO is not statically stable in its longrange order.

First, we observe that the majority of the drop-cast sample is turbostratically multi-layer, with apparent Moire patterns in the few-layer regions (Figure $5 \mathrm{a}-\mathrm{b}$ ). As expected, the presence of functional groups and surface contaminants appears to decouple interactions between the graphitic lattice of adjacent layers ${ }^{15,22}$. Figure $5 \mathrm{~b}$ has been color-coded to represent the number of layers in each region of the image. Second, we show that the underlying structure of the monolayer regions (Figure $5 \mathrm{c}-\mathrm{d}$ ) is nanocrystalline and the $\mathrm{sp}^{2}$ structure of each crystal is independently rotated with respect to each other. This latter observation is a notable addition in potential structures to the previous reports of amorphous (structural models ${ }^{3,13}$ ) and observations of uniform large-area $\mathrm{sp}^{2}$ (TEM results ${ }^{7,15,21,26}$ ) and is characterized quantitatively through Fast Fourier Transforms (FFTs) of regions in the image. The line scans in Figure 5e show increasing sharpness of the polar FFT demonstrating increasing order for smaller regions. 

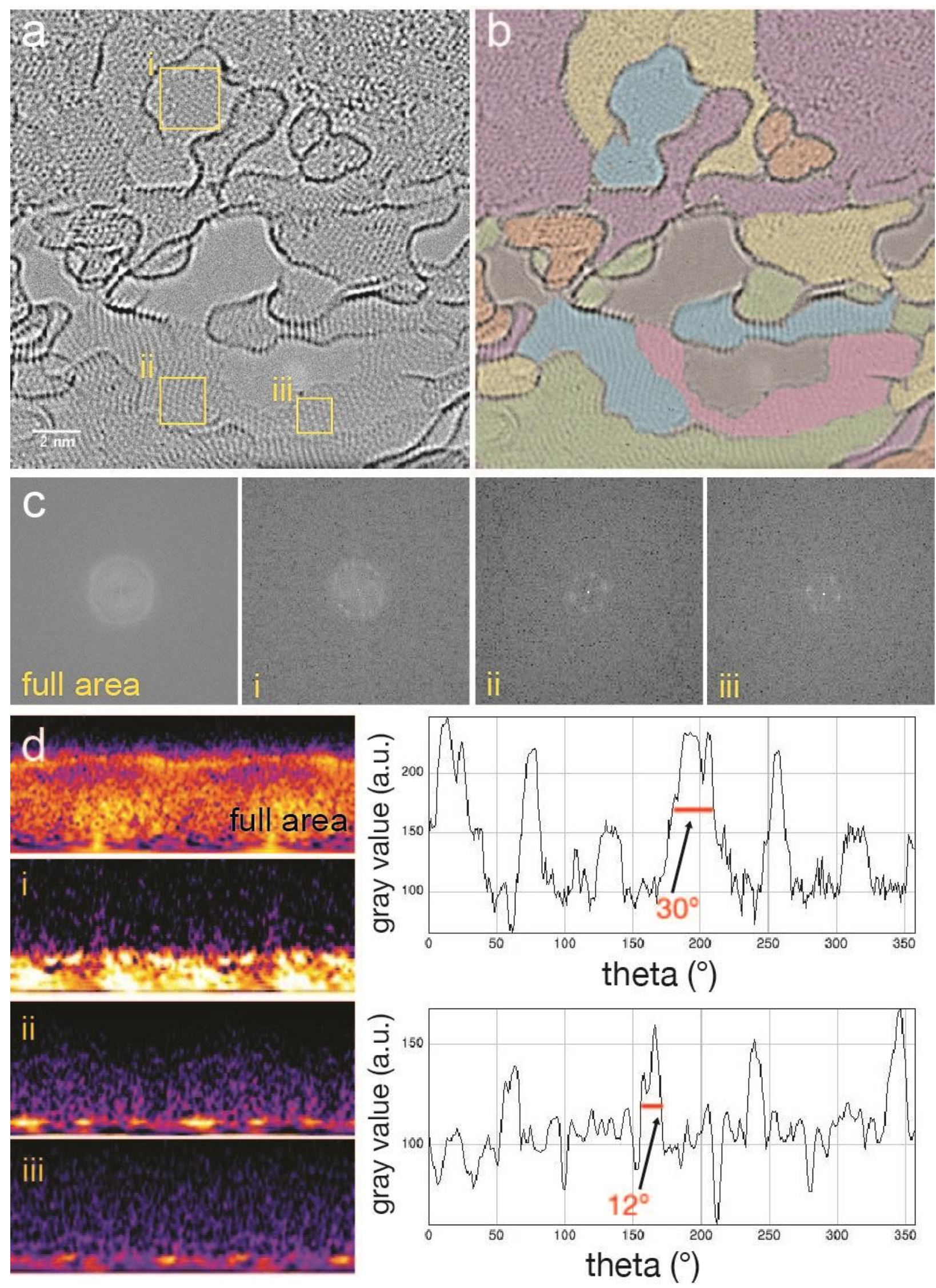
Figure 5 (a) high resolution image of mono- and few- layer grapene oxide. (b) overlayed color represents layers of the material, showing turbostratic stacking and thicker and thinner regions. (c) false color polar transforms of the FFTs (theta X-axis, 1/d y-axis) of the FFTs (d) of regions of the image show an increase in sharpness of the band and points at $1 / \mathrm{d}$ spacing of $1 / 0.19 \mathrm{~nm}^{-1}$ to $1 / 0.20 \mathrm{~nm}^{-1}$ (e) line scans show the increase in sharpness of the lattice points from shows the line scans from regions ii and iii.

The average size of the $\mathrm{sp}^{2}$ nanocrystallites was calculated using around 10 images collected at $700{ }^{\circ} \mathrm{C}$. The boundaries between crystallites are apparent in single-layer areas, but are not obvious in the majority of the regions of shown in Figure 5, in which the material is few or multi-layer. The intra- and inter-layer rotation between crystallites results in a film that appears amorphous in FFTs of regions larger than $\sim 100 \mathrm{~nm}^{2}$, even in thin regions. When an FFT is taken of a much smaller region one to a few crystallites are clear, with the average size of a single crystal of $\sim 2 \mathrm{~nm}^{2}$. We note that the size of the crystalline regions do not change with time at the elevated temperature and under the exposure of the beam in order to rule out heat induced domain size changes. Since mechanically exfoliated graphene from graphitic parent material has larger grain sizes the disorder is likely introduced during the oxidation process, suggesting that tunable transport in rGO derived from chemical exfoliation will remain limited without sufficient temperature for $\mathrm{sp}^{2}$ re-crystallization $\left(>1000^{\circ} \mathrm{C}\right)$.

Evolution of annealed GO In order to decouple the oxygen removal from the surface contaminants, we compare these HRTEM results with spectroscopic data. Unlike graphene, GO undergoes chemical changes upon heating, including carbon removal from lattice site ${ }^{37}$. In addition to the removal of amorphous surface contaminants, oxygen-containing functional groups attached to the basal plane are removed with increasing temperature, as is reported by many others ${ }^{8,25,38}$. Further, simulations show that thermal annealing in the absence of surface 
contaminants reduce the oxygen content of $\mathrm{rGO}^{8,20}$. However here, despite a reduction in oxygen content of $70 \%$ when heated to $700{ }^{\circ} \mathrm{C}$ in a vacuum, cooled, and then analyzed, the correlating images consistently show amorphous material.

To determine whether heating to $700{ }^{\circ} \mathrm{C}$ dramatically changes the nanocrystalline nature of GO, we collected selected area diffraction patterns (SADP) of GO that had not been heated previously with decreasing selected area aperture sizes. We deposited GO on lacey carbon grids and examined the diffraction patterns from GO flakes that were very thin and appeared monolayer and flat in their projection with respect to the electron beam. Figure 6 shows four different aperture sizes: (a) $120 \mu \mathrm{m}$, (b) $50 \mu \mathrm{m}$, (c) $20 \mu \mathrm{m}$ and (d) $10 \mu \mathrm{m}$, and their associated line traces to demonstrate an increasing degree of order with decreasing aperture size (e-h). At the smallest (10 um) aperture, the sample is still apparently multi-crystalline which is qualitatively consistent with the high temperature images.
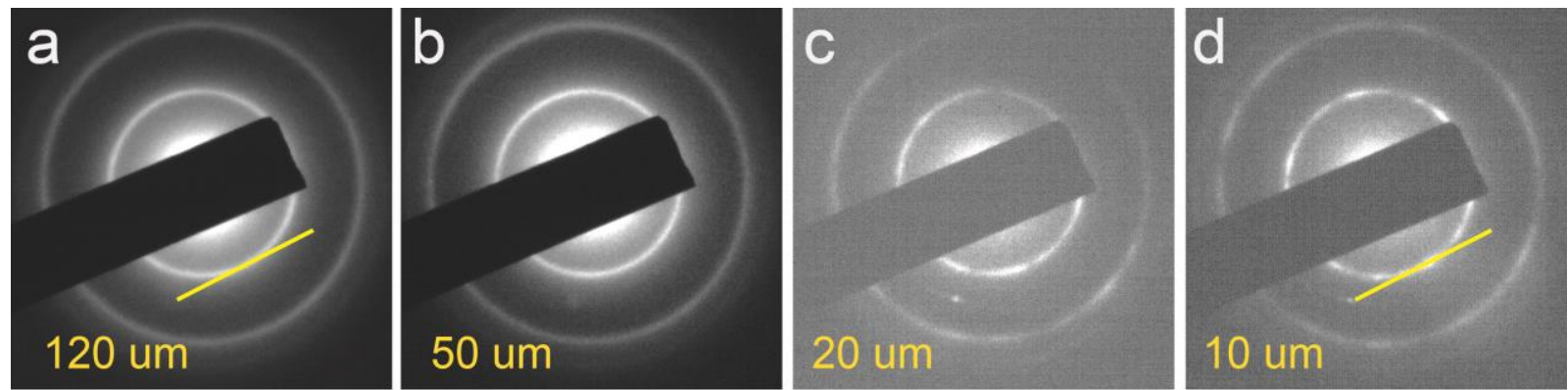

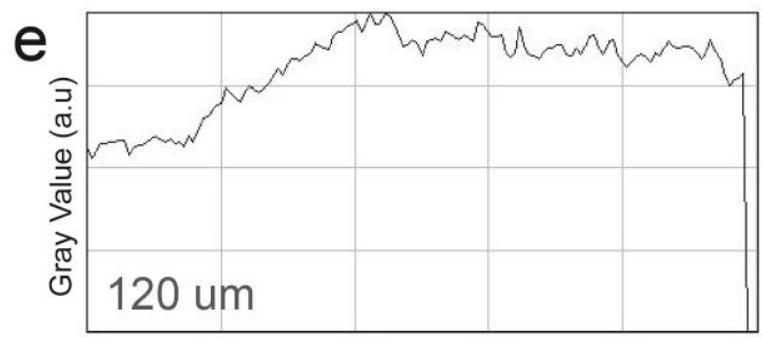

Distance

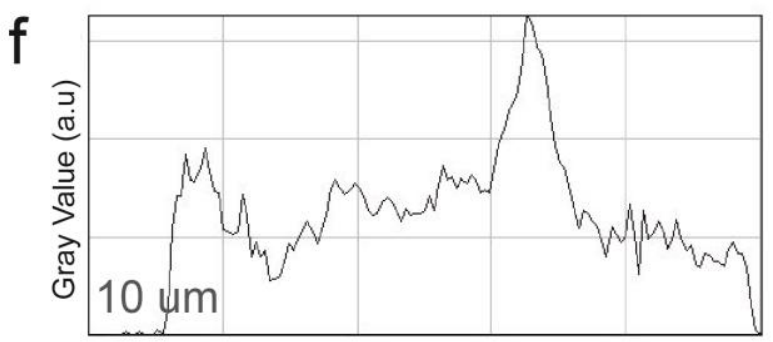

Distance

Figure 6 SADPs for GO collected at room temperature show increasing order with decreasing aperture size (a-d) with line traces showing sharpness of the diffraction pattern (e-h). The sample is multi-crystalline at the smallest aperture size. 
Annealing to $700{ }^{\circ} \mathrm{C}$ most notably, and unsurprisingly, reduces the oxygen content of the GO. The initial carbon to oxygen ratio is observed from XPS to be 1.8:1 (35\% oxygen). The C1s peak (shown in Figure 7a) can be deconvolved into four peaks centered at $285.5 \mathrm{eV}$ (hydrocarbons), $287.4 \mathrm{eV}(\mathrm{C}=\mathrm{O}), 284.6 \mathrm{eV}(\mathrm{C}=\mathrm{C}), 289.2(\mathrm{O}-\mathrm{C}=\mathrm{O})$. Heating increases the carbon to oxygen ratio to 8:1 and shifts the $\mathrm{C} 1$ s peak to deconvolve into $284.4 \mathrm{eV}(\mathrm{C}=\mathrm{C}), 285.5$ (hydrocarbons), $284 \mathrm{eV}$ ( $\mathrm{sp}^{2}$ carbon), and $289.9(\mathrm{O}-\mathrm{C}=\mathrm{O})$, effectively eliminating the $\mathrm{C}=\mathrm{O}$ peak (Figure 7b). The heated samples in the TEM inevitably reduce the oxygen content of the GO. Meanwhile, heating does not change the shape or position of the characteristic $\mathrm{D}\left(1330 \mathrm{~cm}^{-1}\right)$ and $\mathrm{G}\left(1590 \mathrm{~cm}^{-1}\right)$ Raman peak shapes drastically (Figure $\left.7 \mathrm{c}\right)$. Similarly, the $\mathrm{I}_{\mathrm{D}} / \mathrm{I}_{\mathrm{G}}$ ratio changes only slightly from 1.09 to 1.13 (just outside standard error bars) and suggests little change to the crystallite size and that high temperature images are representative of the unheated GO, though defects could be introduced into the lattice upon heating. Moreover, the HRTEM results very well support the models presented for graphene and graphene oxide presented recently ${ }^{39-41}$. 

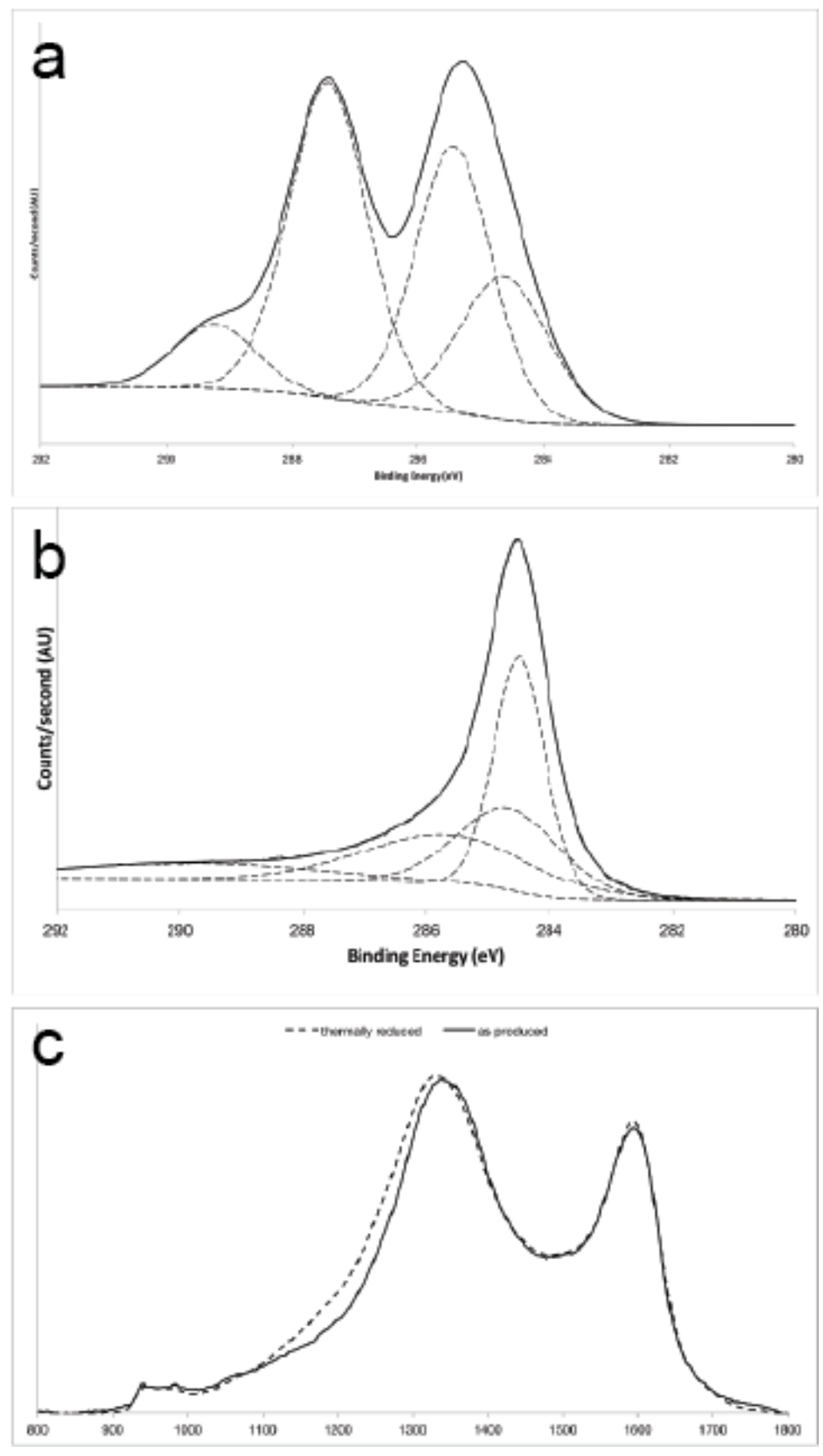

Figure 7 XPS C1s spectra for (a) as-produced and (b) thermally annealed (b) show the change in surface chemical composition of the GO samples. Raman Spectroscopy (c) shows a small change in the $\mathrm{I}_{\mathrm{D}} / \mathrm{I}_{\mathrm{G}}$ ratio but a general consistency in peak shape. 
XPS data were also collected for samples annealed at a range of temperatures to confirm the evolution of oxygen removal (Supplementary Figure 3). In air, the material combusts between $400{ }^{\circ} \mathrm{C}$ and $500{ }^{\circ} \mathrm{C}$, leading first to an increase in oxygen to carbon ratio and then insufficient material to take data. TGA confirms that combustion occurs around $500{ }^{\circ} \mathrm{C}$ (Supplementary Figure 4). As expected, the oxygen content is lower with increasing annealing temperature in vacuum, correlated strongly with a decrease in the $\mathrm{I}_{\mathrm{D}} / \mathrm{I}_{\mathrm{G}}$ ratio.

However, as with prior TGA studies under nitrogen, we observe dehydration of adsorbed water around $100{ }^{\circ} \mathrm{C}$. Also consistent with prior studies, we observe a slight change in slope near 400 ${ }^{\circ} \mathrm{C}$, which we attribute to the release of amorphous surface contaminants rather than oxygencontaining functional groups and carbon from the $\mathrm{sp}^{2}$ lattice. Previous work with Mass Spectroscopy has shown carbon removal at this stage ${ }^{38}$. This change in slope is subtle but repeatable, and consistent with the presence of a thin layer of light elements adsorbed on the surface of the native GO. Further, direct observation of in situ heating shows no significant carbon removal from the lattice even upon extended periods of heating up to $700{ }^{\circ} \mathrm{C}$.

\section{Conclusion}

These results show how using an in-situ heating holder can help elucidate the atomic structure of GO as a function of temperature by removing surface adsorbed amorphous material that inhibits the AC-TEM imaging. Our evidence supports the Dynamic Structural Model described by Dimiev et al. and the Schlolz-Boehm model. The degradation of GO into nanocrystallites has implications in the efforts of restoration of transport mechanisms for electronic applications. The ubiquitous presence of amorphous contaminants on defective $\mathrm{GO}$ at room temperature has 
implications for GO design as a catalyst substrate, enhancing electrical conductivity, or the stable cross-linking of GO sheets for membrane separation processes, revising our interpretation of carbon and oxygen removal, lattice defects, and spectroscopic data. With in-situ heating for TEM, the evolution of the structure graphene oxide is revealed beyond long-range order. The rapid degradation of the $\mathrm{GO}$ sample under electron beam irradiation at $80 \mathrm{kV}$ at room temperature shows that care must be taken when analyzing GO samples by TEM and detecting defects or nanopores that are intrinsic to the material is extremely challenging.

\section{ACKNOWLEDGEMENTS}

We thank Dr. N. Ferralis for the assistance in interpretation of Raman spectroscopy and A. Y. Alsalloum for assistance in acquisition of some Raman spectroscopic data. We also thank Adam Graham of CNS for help with imaging methods. We greatly appreciate funding and support for this research from the Deshpande Center for Technological Innovation at MIT. This work made use of the Center for Nanoscale Systems (CNS), a member of the National Nanotechnology Infrastructure Network (NINN), which is supported by the National Science Foundation under NSF award no. ECS-0335765. CNS is part of Harvard University. JHW thanks the Royal Society for support.

\section{AUTHOR CONTRIBUTIONS}

SHD and JCG conceived the idea, SHD and JHW designed the experiments, which were implemented and carried out by SHD, CG, and AWR. The data was analyzed and the manuscript was written from contributions by SHD, JHW, and JCG. 
The authors declare no competing financial interests. 


\section{METHODS}

Nano platelet graphene oxide (>99\% single layer, average flake size $90 \mathrm{~nm}$ ) was purchased from Graphene Supermarket to represent GO produced at scale and was used for all measurements reported herein. Low magnification TEM shows that the majority of the GO flakes after dispersion in IPA were actually larger (Supplementary Figure 5). Annealing was conducted in a CVD furnace under vacuum ( $<30$ mtorr) to mimic TEM conditions. The heating rate in the CVD was about 1 degree $\mathrm{C}$ per second. Samples were annealed for 1 hour to allow them to achieve steady state, although most chemical changes (oxygen removal) occur within seconds to minutes of reaching temperature. Samples were then evaluated using Raman Spectroscopy, X-Ray Photon Electron Spectroscopy (XPS), and Thermograviometric Analysis (TGA), and Fourier Transform Infrared Spectroscopy (FTIR-ATR) in addition to TEM to monitor evolution during heating. All spectroscopic data was conducted at room temperature and ambient pressure except the XPS, which was collected under vacuum.

\section{Sample Preparation}

GO samples were purchased from Graphene Supermarket (Nano Graphene Oxide) to replicate bulk processing conditions of a modified Hummers' Method. GO was dispersed in IPA and drop cast onto silicon nitride chips for spectroscopic analysis and DENS heating chips for TEM imaging. The heating chips were prepared by introducing a slit in the silicon nitride membrane using focused ion beam for free standing graphene. Annealed samples were thermally treated in a custom built CVD at a pressure of $\sim 5-30$ mtorr (vacuum) and with the ends of the tube open to the ambient environment (air) for 30 minutes to one hour. 
Transmission Electron Microscopy

HRTEM images were collected using a JEOL MCO 2200 aberration-corrected transmission electron microscope operated at an accelerating voltage of $80 \mathrm{kV}$. Data were recorded using a Gatan Ultrascan $4 \mathrm{~K} \times 4 \mathrm{~K}$ CCD camera with $1-2 \mathrm{~s}$ acquisition times and 2 pixel binning.

SADPs were collected using a JEOL 2100 HRTEM operated at an accelerating voltage of 200 $\mathrm{kV}$. Using a higher voltage for SADPs does not damage the GO sample due to lower beam intensity $^{17}$.

Image Processing

Processing of TEM images was conducted using ImageJ. Bandpass filters (1-100 pixels) were used to remove uneven illumination on all presented images. Line profiles and false-color images were generated using a Polar Transformer plugin for ImageJ to process FFTs. False color images are LUT Fire in ImageJ.

\section{In situ Heating Holder}

We used a commercially available in situ heating holder from DENS Solutions (SH30-4M-FS). Heating the sample was achieved by passing a current through a platinum resistive coil imbedded in the TEM chip (DENS Solutions DENS-C-30). The resistance of the platinum coil is monitored in a four-point configuration, and the temperature is calculated using the Callendar-Van Dusen equation (with calibration constants provided by the manufacturer). Slits were introduced into the Silicon Nitride film of the TEM chips using a Zeiss NVision Focussed Ion Beam.

Raman Spectroscopy 
Micro Raman spectra was collected using Horiba LabRAM 800 HR spectrometer equipped with He-Ne (632.817) laser and no filter. Collection time was 5 seconds, average 5-10 times; spot size was $\sim 800 \mathrm{~nm}$ in diameter and has a power of $<1 \mathrm{~mW}$ at the sample surface and was corrected for fluorescence. Data was collected across the thin film on 6-1 points and averaged to observe film uniformity and reduce error bars. All graphs include standard error bars. Data was analyzed using LabSpec and peak intensity (area) was used for the calculation of the $\mathrm{I}_{\mathrm{D}} / \mathrm{I}_{\mathrm{G}}$ ratio.

\section{X-Ray Photoelectron Spectroscopy}

XPS data was collected using a Thermofischer Scientific K-Alpha with Aluminum Ka radiation. In order to reduce surface charging, XPS data was collected with the flood gun, which consists of low energy electrons and ions that neutralize the surface of the GO. The XPS samples were prepared with a dilute solution of GO (in IPA) drop cast onto silicon nitride. The use of silicon nitride is to eliminate any contribution of oxidation of the substrate to the peaks. The samples are taken with a 400 um spot size on regions that appear visually homogeneous. Finally, the survey spectra detects the substrate, which indicates that the film is thin, further helping to suppress charging. Data was analyzed using CasaXPS survey spectra to calculate oxygen concentration.

\section{FTIR-ATR Spectroscopy}

Fourier Transform Infrared Spectroscopy was conducted using a Germanium attenuated total reflectance (ATR) crystal in a Thermo Fisher FTIR6700 at ambient conditions. Background and sample scans were taken at a sample rate of 64 .

Thermograviometric Analysis 
TGA data was collected using TA Instruments Q500 in air and nitrogen as an inert environment with about $70 \mathrm{mg}$ of GO. The heating rate was $10 \mathrm{C} / \mathrm{min}$. Data was analyzed using Universal Analysis 2000.

\section{References}

1. Brodie, B. C. On the Atomic Weight of Graphite. Philosophical Transactions of the Royal Society of London 1859, 149, 249-259.

2. Rourke, J. P.; Pandey, P. A.; Moore, J. J.; Bates, M.; Kinloch, I. A.; Young, R. J.; Wilson, N. R. The Real Graphene Oxide Revealed: Stripping the Oxidative Debris From the Graphene-Like Sheets. Angew. Chem. Int. Ed. 2011, 50, 3173-3177.

3. Robertson, A. W.; Ford, C.; He, K.; Kirkland, A. I.; Watt, A. A. R.; Warner, J. H. PbTe Nanocrystal Arrays on Graphene and the Structural Influence of Capping Ligands. Chemistry of Materials 2014, 26, 1567-1575.

4. Hummers, W. S.; Offeman, R. E. Preparation of Graphitic Oxide. 1958, 1-1.

5. Dimiev, A. M.; Alemany, L. B.; Tour, J. M. Graphene Oxide. Origin of Acidity, Its Instability in Water, and a New Dynamic Structural Model. ACS Nano 2013, 7, 576-588.

6. Dimiev, A. M.; Polson, T. A. Contesting the Two-Component Structural Model of Graphene Oxide and Reexamining the Chemistry of Graphene Oxide in Basic Media. CARBON 2015, 93, 544-554.

7. Gómez-Navarro, C.; Meyer, J. C.; Sundaram, R. S.; Chuvilin, A.; Kurasch, S.; Burghard, M.; Kern, K.; Kaiser, U. Atomic Structure of Reduced Graphene Oxide. Nano Lett. 2010, 10, 1144-1148.

8. $\quad$ Bagri, A.; Mattevi, C.; Acik, M.; Chabal, Y. J.; Chhowalla, M.; Shenoy, V. B. Structural Evolution During the Reduction of Chemically Derived Graphene Oxide. Nature Publishing Group 2010, 2, 581-587.

9. $\quad$ Li, Z.; Wang, Y.; Kozbial, A.; Shenoy, G.; Zhou, F.; McGinley, R.; Ireland, P.; Morganstein, B.; Kunkel, A.; Surwade, S. P.; et al. Effect of Airborne Contaminants on the Wettability of Supported Graphene and Graphite. Nature Materials 2013, 12, 1-8.

10. Bonanni, A.; Ambrosi, A.; Chua, C. K.; Pumera, M. Oxidation Debris in Graphene Oxide Is Reponsible for Its Inherent Electroactivity. ACS Nano 2014, 8, 4197-4204.

11. Li, Z.; Kozbial, A.; Nioradze, N.; Parobek, D.; Shenoy, G. J.; Salim, M.; Amemiya, S.; Li, L.; Liu, H. Water Protects Graphitic Surface From Airborne Hydrocarbon Contamination. ACS Nano 2015, acsnano.5b04843.

12. Li, X.; Yang, X.; Jia, L.; Ma, X.; Zhu, L. Carbonaceous Debris That Resided in Graphene Oxide/Reduced Graphene Oxide Profoundly Affect Their Electrochemical Behaviors. Electrochemistry Communications 2012, 23, 94-97.

13. Lerf, A.; He, H.; Forster, M.; Klinoski, J. Structure of Graphite Oxide Revisited. J. Phys. Chem. B 1998, 102, 4477-4482.

14. Larciprete, R.; Fabris, S.; Sun, T.; Lacovig, P.; Baraldi, A.; Lizzit, S. Dual Path Mechanism in the Thermal Reduction of Graphene Oxide. J. Am. Chem. Soc. 2011, 133, 17315-17321. 
15. Erickson, K.; Erni, R.; Lee, Z.; Alem, N.; Gannett, W.; Zettl, A. Determination of the Local Chemical Structure of Graphene Oxide and Reduced Graphene Oxide. Adv. Mater. 2010, 22, 4467-4472.

16. Eigler, S.; Dotzer, C.; Hirsch, A.; Enzelberger, M.; Müller, P. Formation and Decomposition of CO 2Intercalated Graphene Oxide. Chemistry of Materials 2012, 24, 1276-1282.

17. Wilson, N. R.; Pandey, P. A.; Beanland, R.; Young, R. J.; Kinloch, I. A.; Gong, L.; Liu, Z.; Suenaga, K.; Rourke, J. P.; York, S. J.; et al. Graphene Oxide: Structural Analysis and Application as a Highly Transparent Support for Electron Microscopy. ACS Nano 2009, 3, 2547-2556.

18. Pantelic, R. S.; Meyer, J. C.; Kaiser, U.; Baumeister, W.; Plitzko, J. M. Graphene Oxide: a Substrate for Optimizing Preparations of Frozen-Hydrated Samples. Journal of Structural Biology 2010, 170, 152-156.

19. Peplow, M. Graphene Booms in Factories but Lacks a Killer App. Nature 2015, 522, 268269.

20. Lin, L.-C.; Grossman, J. C. Atomistic Understandings of Reduced Graphene Oxide as an Ultrathin-Film Nanoporous Membrane for Separations. Nature Communications 2015, 6, $1-7$.

21. Algara-Siller, G.; Lehtinen, O.; Turchanin, A.; Kaiser, U. Dry-Cleaning of Graphene. Appl. Phys. Lett. 2014, 104, 153115.

22. Eda, G.; Chhowalla, M. Chemically Derived Graphene Oxide: Towards Large-Area ThinFilm Electronics and Optoelectronics. Adv. Mater. 2010, 22, 2392-2415.

23. Loh, K. P.; Bao, Q.; Eda, G.; Chhowalla, M. Graphene Oxide as a Chemically Tunable Platform for Optical Applications. Nature Publishing Group 2010, 2, 1015-1024.

24. Dreyer, D. R.; Park, S.; Bielawski, C. W.; Ruoff, R. S. The Chemistry of Graphene Oxide. Chem. Soc. Rev. 2009, 39, 228.

25. Mattevi, C.; Eda, G.; Agnoli, S.; Miller, S.; Mkhoyan, K. A.; Celik, O.; Mastrogiovanni, D.; Granozzi, G.; Garfunkel, E.; Chhowalla, M. Evolution of Electrical, Chemical, and Structural Properties of Transparent and Conducting Chemically Derived Graphene Thin Films. Adv. Funct. Mater. 2009, 19, 2577-2583.

26. Pantelic, R. S.; Meyer, J. C.; Kaiser, U.; Baumeister, W.; Plitzko, J. M. Graphene Oxide: a Substrate for Optimizing Preparations of Frozen-Hydrated Samples. Journal of Structural Biology 2010, 170, 152-156.

27. Szabó, T.; Berkesi, O.; forgo, P.; josepovits, K.; sanakis, Y.; petridis, D.; Dékány, I. Evolution of Surface Functional Groups in a Series of Progressively Oxidized Graphite Oxides. Chemistry of Materials 2006, 18, 2740-2749.

28. Feng, H.; Cheng, R.; Zhao, X.; Duan, X.; Li, J. A Low-Temperature Method to Produce Highly Reduced Graphene Oxide. Nature Communications 1AD, 4, 1539-7.

29. Eda, G.; Fanchini, G.; Chhowalla, M. Large-Area Ultrathin Films of Reduced Graphene Oxide as a Transparent and Flexible Electronic Material. Nature Nanotechnology 2008, 3, 270-274.

30. Girit, C.; Meyer, J.; Erni, R.; Rossell, M. D.; Kisielowski, C.; Yang, L.; Park, C.-H.; Crommie, M. F.; Cohen, M.; Louie, S.; et al. Graphene at the Edge: Stability and Dynamics. Science 2009, 323, 1705-1708.

31. Warner, J. H.; Margine, E. R.; Mukai, M.; Robertson, A. W.; Giustino, F.; Kirkland, A. I. Dislocation-Driven Deformations in Graphene. Science 2012, 337, 209-212. 
32. Bell, D. C.; Russo, C. J.; Benner, G. Sub-Ångstrom Low-Voltage Performance of a Monochromated, Aberration-Corrected Transmission Electron Microscope. Microsc Microanal 2010, 16, 386-392.

33. Mkhoyan, K. A.; Contryman, A. W.; Silcox, J.; Stewart, D. A.; Eda, G.; Mattevi, C.; Miller, S.; Chhowalla, M. Atomic and Electronic Structure of Graphene-Oxide. Nano Lett. 2009, 9, 1058-1063.

34. He, K.; Robertson, A. W.; Gong, C.; Allen, C. S.; Xu, Q.; Zandbergen, H.; Grossman, J. C.; Kirkland, A. I.; Warner, J. H. Controlled Formation of Closed-Edge Nanopores in Graphene. Nanoscale 2015.

35. Casabianca, L. B.; Shaibat, M. A.; Cai, W. W.; Park, S.; Piner, R.; Ruoff, R. S.; Ishii, Y. NMR-Based Structural Modeling of Graphite Oxide Using Multidimensional 13C SolidState NMR and Ab Initio Chemical Shift Calculations. J. Am. Chem. Soc. 2010, 132, 5672-5676.

36. Thomas, H. R.; Day, S. P.; Woodruff, W. E.; Vallés, C.; Young, R. J.; Kinloch, I. A.; Morley, G. W.; Hanna, J. V.; Wilson, N. R.; Rourke, J. P. Deoxygenation of Graphene Oxide: Reduction or Cleaning? Chemistry of Materials 2013, 25, 3580-3588.

37. Eigler, S.; Grimm, S.; Hirsch, A. Investigation of the Thermal Stability of the Carbon Framework of Graphene Oxide. Chem. Eur. J. 2013, 20, 984-989.

38. Chen, C.-M.; Zhang, Q.; Yang, M.-G.; Huang, C.-H.; Yang, Y.-G.; Wang, M.-Z. Structural Evolution During Annealing of Thermally Reduced Graphene Nanosheets for Application in Supercapacitors. CARBON 2012, 50, 3572-3584.

39. Cançado, L. G.; Jorio, A.; Ferreira, E. H. M.; Stavale, F.; Achete, C. A.; Capaz, R. B.; Moutinho, M. V. O.; Lombardo, A.; Kulmala, T. S.; Ferrari, A. C. Quantifying Defects in Graphene via Raman Spectroscopy at Different Excitation Energies. Nano Lett. 2011, 11, 3190-3196.

40. Lucchese, M. M.; Stavale, F.; Ferreira, E. H. M.; Vilani, C.; Moutinho, M. V. O.; Capaz, R. B.; Achete, C. A.; Jorio, A. Quantifying Ion-Induced Defects and Raman Relaxation Length in Graphene. CARBON 2010, 48, 1592-1597.

41. Eigler, S.; Hof, F.; Enzelberger-Heim, M.; Grimm, S.; Müller, P.; Hirsch, A. Statistical Raman Microscopy and Atomic Force Microscopy on Heterogeneous Graphene Obtained After Reduction of Graphene Oxide. J. Phys. Chem. C 2014, 118, 7698-7704. 